\title{
LINEARLY FIRST- AND SECOND-ORDER, UNCONDITIONALLY ENERGY STABLE SCHEMES FOR THE PHASE FIELD CRYSTAL MODEL
}

\author{
XIAOFENG YANG ${ }^{\ddagger \star}$ AND DAOZHI HAN ${ }^{\dagger}$
}

\begin{abstract}
In this paper, we develop a series of linear, unconditionally energy stable numerical schemes for solving the classical phase field crystal model. The temporal discretizations are based on the first order Euler method, the second order backward differentiation formulas (BDF2) and the second order Crank-Nicolson method, respectively. The schemes lead to linear elliptic equations to be solved at each time step, and the induced linear systems are symmetric positive definite. We prove that all three schemes are unconditionally energy stable rigorously. Various classical numerical experiments in $2 \mathrm{D}$ and $3 \mathrm{D}$ are performed to validate the accuracy and efficiency of the proposed schemes.
\end{abstract}

\section{INTRODUCTION}

Crystallization is a well-known technique in the purification of solid compounds. If a saturated hot solution is allowed to cool off, the solute is no longer soluble in the solvent and forms crystals of pure compound. During this process of crystal growth that is the major stage in crystallization, the atoms, ions or molecules are rearranged into a highly ordered crystalline structure. Such a process is a typical example of phase transitions in which the mass transfer of a solute from the liquid solution or vapor phase to a solid crystalline phase happens. While the crystal grows, certain defects, such as vacancies, grain boundaries, and dislocations, may form due to growth disturbance as impurities are excluded from the growing crystals. This also explains why real crystals are never perfect. The crystals always contain a considerable density of defects and imperfections that affect their physical, chemical, mechanical and electronic properties. The existence of defects also plays an important role in various technological processes and phenomena such as annealing, precipitation, diffusion, oxidation and others.

To understand the process of crystal growth, many mathematical models have been developed, and numerical simulations based on these models have been carried out (cf. [14,19,20] and the references therein). Among these models, the phase-field method is widely adopted [6,7], where the crystal/liquid interface is considered as a transition layer over which a continuous, but steep change of some physical quantities (such as concentration, density, magnetization, etc.) occur. The two-phase medium is then described by some continuous phase field variables. The phasefield models can be naturally derived from variational principles, i.e., via minimizing the free

Key words and phrases. Phase-field Crystal; Second Order; Linear Scheme; Cahn-Hilliard; Unconditional Energy Stability.

$\ddagger^{*}$ Corresponding author, Department of Mathematics, University of South Carolina, Columbia, SC 29208, USA; Email: xfyang@math.sc.edu.

$\dagger$ Department of Mathematics, Indiana University at Bloomington; Email: djhan@iu.edu. 
energy of the total system. As a result, the derived system satisfies an energy dissipation law, which justifies its thermodynamic consistency and leads to a mathematically well-posed model. Moreover, the presence of the energy law serves as a guideline for the design of energy stable numerical schemes. The idea of phase field approach dates back to the pioneering work of [23] and [30]. It has been successfully employed in many fields of science and engineering ever since. The phase field method now becomes one of the major modeling and computational tools for the study of interfacial phenomena (cf. $[1,10,13,15,17,18,27,34]$, and the references therein).

In $[6,7]$, the authors had developed a very simple phase field crystal (PFC) model that showed great versatility to study the dynamics of atomic-scale crystal growth on diffusive time scales. An order parameter (phase field variable) that represents the concentration field of a coarse-grained temporal average of the density of atoms, is introduced to describe the phase transition from the liquid phase to the crystal phase. More precisely, the density is relatively homogeneous in the liquid phase and spatially periodic (i.e., crystalline) in the solid phase. Thus, a free energy is postulated to generate the periodic structure of a crystal lattice, by incorporating a specific form of the spatial gradients,. The model is flexible and can be applied to simulate various phenomena, for instances, epitaxial growth, material hardness, grain growth, reconstructive phase transitions, and crack propagations.

From the numerical point of view, to solve the phase field system, it is especially desirable to design numerical schemes that preserve the energy dissipation law at the discrete level. On the one hand, the preservation of the energy law is critical for the numerical schemes to capture the correct long time dynamics of the system. On the other hand, the unconditional stability of the energy-law preserving schemes provides flexibility for dealing with the stiffness issue in phase-filed models. In particular, the dynamics of the coarse-graining (macroscopic) process may undergo rapid changes near the interface, so the non-compliance of energy dissipation laws may lead to spurious numerical solutions if the grid and time step sizes are not carefully chosen.

We notice that there have been some numerical analysis works on the development of energy stable schemes for the solving the PFC model recently. In [24,31], the authors propose a first-order and a second-order numerical schemes for solving the PFC model based on the convex splitting approach. Both schemes satisfy modified energy laws and therefore unconditionally stable. However, the convex-splitting method typically leads to nonlinear equations to be solved at each time step, cf. $[12,29]$. One needs to design some efficient iterative solvers for solving the nonlinear system. In [8], the authors present a nonlinear numerical scheme, where the nonlinear potential is discretized by a quadrature formula. Though the scheme is shown to be unconditionally energy stable, there is no theoretical result on the solvability of the nonlinear system. In relation to solving the PFC model, we point out that a general review of the existing numerical methods and their stability and solvability for the Cahn-Hilliard equation is given in [29].

Therefore, our focus in this article is to develop some efficient numerical schemes that preserve the energy dissipation law as the PDE system. In particular, the nonlinear term in the model is linearized by using the so-called Lagrangian-Multiplier approach, a technique that is originally introduced to discretize the double well potential in the fourth order Cahn-Hilliard equation [9]. Based on this linearization, we propose three time discretization schemes according to the first 
order Euler method, the second order backward differentiation formulas (BDF2) and the second order Crank-Nicolson method, respectively. We show that our schemes satisfy modified energy laws and are unconditionally energy stable. Moreover, at each time step, the schemes lead to linear elliptic equations, where the linear operators are symmetric positive definite that can be solved efficiently using existing fast solvers. Thus, the developed schemes can perform significantly better than other nonlinear schemes. Various 2D and 3D numerical results are presented to validate the accuracy and efficiency of our schemes.

The rest of the paper is organized as follows. In Section 2, we present the PFC model and the associated energy law. In Section 3, we develop the numerical schemes and prove their unconditional stability and unconditionally unique solvability in the time discrete case. in Section 4, We present some numerical results to demonstrate the accuracy and efficiency of our numerical schemes. Some concluding remarks are presented in Section 5.

\section{GOVERNING SYSTEMS}

We now introduce the PFC model as is proposed in $[6,7]$ that describes the phenomena of crystal growth on the atomic length and diffusive time scales. The model not only incorporates the elastic and plastic deformations in a natural manner, but also enables access to time scales much larger than conventional atomic methods. The predictions yielded by the model have been shown to be consistent with the observations in epitaxial growth, material hardness, grain boundary growth, reconstructive phase transitions, and crack propagation in ductile materials [6-8].

Let $a, \eta$, be two positive constants such that $\eta<a$ and $\eta \ll 1$. A phase field variable $\phi: \Omega \subset$ $R^{d}(d=2,3) \rightarrow R$ is introduced to describe the local atomic density field. The free energy of the system is defined as follows

$$
E(\phi)=\int_{\Omega}\left(\frac{1}{4} \phi^{4}+\frac{a-\eta}{2} \phi^{2}-a|\nabla \phi|^{2}+\frac{1}{2}(\Delta \phi)^{2}\right) d \boldsymbol{x},
$$

where, $\nabla$ and $\Delta$ are the gradient and Laplace operators, respectively. Such a free energy functional also arises in many other physical systems [16,21,25,28].

The dynamic equations can be derived as a gradient flow of the energy functional (2.1) in certain metric. Taking the variational derivative of (2.1) in the negative norm gives the conservative dynamic equations

$$
\begin{aligned}
& \phi_{t}=\nabla \cdot(M(\phi) \nabla \mu), \\
& \mu=\phi^{3}+(a-\eta) \phi+2 a \Delta \phi+\Delta^{2} \phi,
\end{aligned}
$$

where $M(\phi)$ is the mobility function, $\mu:=\frac{\delta E(\phi)}{\delta \phi}$ is called the chemical potential. Here we assume that the mobility function is a function of the order parameter such that $M(\phi) \geq m>0$ for a fixed constant $m$. For instance, one can take $M(\phi)=\sqrt{\phi^{2}(\phi-1)^{2}+\eta^{2}}$ or simply, a constant $\eta$. We impose either the periodic boundary conditions or the following boundary conditions

$$
\left.\partial_{\mathbf{n}} \phi\right|_{\partial \Omega}=0,\left.\quad \partial_{\mathbf{n}}(\Delta \phi)\right|_{\partial \Omega}=0,\left.\quad \partial_{\mathbf{n}}\left(\Delta^{2} \phi\right)\right|_{\partial \Omega}=0,
$$

where $\mathbf{n}$ is the unit outward normal on the boundary $\partial \Omega$. 
We note that the boundary conditions (2.4) imply the no-flux boundary condition $\left.\partial_{\mathbf{n}} \mu\right|_{\partial \Omega}=0$. It follows that the PFC model (2.2) and (2.3) is mass-conservative, in the sense that $\frac{d}{d t} \int_{\Omega} \phi d x=0$. Moreover, by taking the $L^{2}$ inner product of (2.2) with $\mu$, utilizing (2.3), performing integration by parts, one finds that the system satisfy an energy law

$$
\frac{d}{d t} E(\phi)=-\|\sqrt{M(\phi)} \nabla \mu\|^{2} \leq 0 .
$$

In the sequel, we will carefully design temporal discrete schemes which satisfies the deiscrete version of the continuous energy law (2.5).

Before we present our numerical methods, we remark that a simpler alternative model of Allen-Cahn type can be derived by taking the variational derivative of $(2.1)$ in the $L^{2}$ sense. The governing equation becomes

$$
\phi_{t}=-M(\phi) \mu,
$$

where $\mu$ takes the same form as (2.3). The boundary conditions are imposed as $\left.\partial_{\mathbf{n}} \phi\right|_{\partial \Omega}=0$ and $\left.\partial_{\mathbf{n}}(\Delta \phi)\right|_{\partial \Omega}=0$. One can check that (2.6) satisfies an energy-conservation law

$$
\frac{d}{d t} E(\phi)=-\|\sqrt{M(\phi)} \mu\|^{2} \leq 0 .
$$

However, (2.6) does not conserve mass. To restore the property of mass-conservation, one can take the functional derivative with the constraint $\int_{\Omega} \phi d x=c$, where $c$ is a constant, so that a non-local Lagrange multiplier will arise in the equation (cf. [33]). Another approach is to add a penalty term to the free energy functional (2.1) to enforce the mass-conservation (cf. [5]). Our numerical methods will be equally applicable to the conservative version of (2.6). From now on, we shall concentrate on solving the system (2.2) and (2.3).

\section{NumericAl Schemes}

We now construct several discrete-in-time numerical schemes for solving the PFC model (2.2) -(2.3) and establish their energy stabilities. It will be clear that the energy stabilities of the semidiscrete schemes are also valid in the fully discrete formulation, for instance by finite element or spectral spatial discretizations.

The major difficulty for the design of energy stable schemes lies in the treatment of the nonlinear term $\phi^{3}$ in (2.3). First, it appears not feasible to treat this nonlinear term explicitly as the stabilized approach in $[26,32]$ because the maximum principle does not hold for the PDE system (2.2)-(2.3). Thus similar to the time-discretization schemes for Cahn-Hilliard type equations, Wang et. al. in $[24,31]$ derive energy stable schemes based on the convex splitting approach, where this term is treated implicitly. Hence nonlinear schemes are developed in their work.

We aim to obtain some more effective numerical schemes, in particular, the linear schemes. Inspired by the Lagrange-Multiplier approach to discretize the cubic polynomial term induced from the double well potential for the Cahn-Hilliard model [9], we introduce two auxiliary functions

$$
U=\phi^{2}, \psi=\Delta \phi
$$


Using the variables $\phi, U, \psi$, we recast the energy functional (2.1) as

$$
E(\phi, U, \psi)=\int_{\Omega}\left(\frac{1}{4} U^{2}+\frac{a-\eta}{2} \phi^{2}-a|\nabla \phi|^{2}+\frac{1}{2} \psi^{2}\right) d x
$$

In turn, we have the equivalent PDE system as follows.

$$
\begin{aligned}
& \phi_{t}=\nabla \cdot(M(\phi) \nabla \mu), \\
& \mu=U \phi+(a-\eta) \phi+2 a \Delta \phi+\Delta \psi, \\
& \psi=\Delta \phi,
\end{aligned}
$$

by taking the time derivative of $U$, we obtain an ordinary differential equation

$$
U_{t}=2 \phi \phi_{t}
$$

Therefore, the system (3.3)-(3.4)-(3.5)-(3.6) form a closed PDE system for the variables $\phi, \mu, \psi, U$. Since (3.6) is an ODE system, thus $U$ does not need any boundary conditions. The boundary conditions can be either periodic or of Neumann type as follows.

$$
\left.\partial_{n} \phi\right|_{\partial \Omega}=0,\left.\quad \partial_{n} \mu\right|_{\partial \Omega}=0,\left.\quad \partial_{n} \psi\right|_{\partial \Omega}=0 .
$$

And the the initial conditions are

$$
\phi(t=0)=\phi_{0}, U(t=0)=\phi_{0}^{2} .
$$

We note that the new boundary conditions (3.7) are equivalent to the original boundary conditions (2.4), in view of the strong formulation of (3.3)-(3.5).

The new system (3.3)-(3.6) in terms of the new variables still follows an energy dissipative law. We formally take the $L^{2}$ inner product of (3.3) with $-\mu$, of (3.4) with $\phi_{t}$, of (3.6) with $-\frac{1}{2} U$, and perform integration by parts

$$
\begin{aligned}
-\left(\phi_{t}, \mu\right) & =(M(\phi) \nabla \mu, \nabla \mu), \\
\left(\mu, \phi_{t}\right) & =\left(U \phi, \phi_{t}\right)+(a-\eta)\left(\phi, \phi_{t}\right)-2 a\left(\nabla \phi, \nabla \phi_{t}\right)-\left(\nabla \psi, \nabla \phi_{t}\right), \\
-\frac{1}{2}\left(U_{t}, U\right) & =-\left(\phi \phi_{t}, U\right) .
\end{aligned}
$$

Next, we differentiate (3.5) in time and then take the $L^{2}$ inner product of the resulting equation with $-\psi$

$$
-\left(\psi_{t}, \psi\right)=\left(\nabla \phi_{t}, \nabla \psi\right)
$$

Combinning (3.9)-(3.12), we obtain the new energy dissipation law as follows.

$$
\frac{d}{d t} E(\phi, U, \psi)=-\|\sqrt{M(\phi)} \nabla \mu\|^{2} \leq 0 .
$$

Remark 3.1. We emphasize that the new transformed system (3.3)-(3.4)-(3.5)-(3.6) is exactly equivalent to the original system (2.2)-(2.3) since $U=\phi^{2}$ can be easily obtained by integrating (3.6) with respect to the time.

We will develop numerical schemes for time stepping of the transformed system (3.3)-(3.4)(3.5)-(3.6) which follows the new energy dissipation law (3.13) instead of the energy law (2.5) of the original system (2.2)-(2.3). The proof of the unconditional stability of the schemes follow the 
same lines as in the derivation of the energy law (3.13). Moreover, it is obvious that, for the time continuous case, the energy law (3.13) for the transformed system is exactly the same as the energy law (2.5) for the original system.

We fix some notations here. Let $\delta t>0$ be a time step size and set $t^{n}=n \delta t$ for $0 \leq n \leq N=$ $[T / \delta t]$. We denote by $(f(x), g(x))=\left(\int_{\Omega} f(x) g(x) d x\right)^{\frac{1}{2}}$ the $L^{2}$ inner product between functions $f(x)$ and $g(x)$, and by $\|f\|=\sqrt{(f, f)}$ the $L^{2}$ norm of the function $f(x)$.

3.1. The first-order scheme. A first order scheme for solving the system (3.3)-(3.5) can be readily derived by the backward Euler's method. Having computed $\phi^{n-1}, U^{n-1}$, we compute $\phi^{n+1}, U^{n+1}$ as follows.

$$
\begin{aligned}
& \frac{\phi^{n+1}-\phi^{n}}{\delta t}=\nabla \cdot\left(M\left(\phi^{n}\right) \nabla \mu^{n+1}\right), \\
& \mu^{n+1}=U^{n+1} \phi^{n}+(a-\eta) \phi^{n+1}+2 a \Delta \phi^{n}+\Delta \psi^{n+1}, \\
& \frac{U^{n+1}-U^{n}}{\delta t}=2 \phi^{n} \frac{\phi^{n+1}-\phi^{n}}{\delta t}, \\
& \psi^{n+1}=\Delta \phi^{n+1} .
\end{aligned}
$$

Since (3.16) is a simple algebraic equation, we can rewrite it as

$$
U^{n+1}=U^{n}-2\left(\phi^{n}\right)^{2}+2 \phi^{n} \phi^{n+1} .
$$

In turn, the scheme can be rewritten as

$$
\begin{aligned}
& \frac{\phi^{n+1}-\phi^{n}}{\delta t}=\nabla \cdot\left(M\left(\phi^{n}\right) \nabla \mu^{n+1}\right), \\
& \mu^{n+1}=P_{1}\left(\phi^{n+1}\right)+\Delta \psi^{n+1}+\alpha_{1}, \\
& \psi^{n+1}=\Delta \phi^{n+1} .
\end{aligned}
$$

where

$$
P_{1}(\phi)=2\left(\phi^{n}\right)^{2} \phi+(a-\eta) \phi, \alpha_{1}=U^{n} \phi^{n}-2\left(\phi^{n}\right)^{3}+2 a \Delta \phi^{n} .
$$

Therefore, we can solve for $\phi^{n+1}, \mu^{n+1}, \psi^{n+1}$ directly from (3.19), (3.20) and (3.21). Once we obtain $\phi^{n+1}$, then $U^{n+1}$ will be automatically given by (3.18). That means the new variable $U$ does not involve any extra computational costs.

One can immediately notice that the linear operator $P(\phi)$ is symmetric positive definite, this is because

$$
\left(P_{1}(\phi), \psi\right)=(\phi, P(\psi))
$$

and

$$
\left(P_{1}(\phi), \phi\right) \geq 2\left(\left(\phi^{n}\right)^{2}, \phi^{2}\right)+(a-\eta)\|\phi\|^{2} \geq 0 .
$$

The "=" is valid if and only if $\phi=0$.

Moreover, we have the following theorem for schemes (3.19)-(3.20)-(3.21). 
Theorem 3.1. When the mobility parameter is a constant, $M(\phi)=M$, the scheme (3.19)(3.20)-(3.21) leads to a symmetric (self-adjoint) positive definite linear equation for the variable $\phi^{n+1}$.

Proof. From (3.19), we have

$$
\int_{\Omega} \phi^{n+1} d x=\int_{\Omega} \phi^{n} d x=\cdots=\int_{\Omega} \phi^{0} d x
$$

Let $\alpha_{\phi}=\frac{1}{|\Omega|} \int_{\Omega} \phi^{0} d x, \beta_{\mu}=\frac{1}{|\Omega|} \int_{\Omega} \mu^{n+1} d x$, and we define

$$
\widehat{\phi}^{n+1}=\phi^{n+1}-\alpha_{\phi}, \widehat{\mu}^{n+1}=\mu^{n+1}-\beta_{\mu} .
$$

It follows from Eq. (3.20) that $\beta_{\mu}=\frac{1}{|\Omega|} \int_{\Omega} P_{1}\left(\phi^{n+1}\right)+\left(U^{n}-2\left(\phi^{n}\right)^{2}\right) \phi^{n} d x$. Thus, the scheme (3.19)-(3.20)-(3.21) implies that $\widehat{\phi}^{n+1}$ and $\widehat{\mu}^{n+1}$ are the solutions to the following equations,

$$
\begin{aligned}
& \frac{\phi}{\delta t}-M \Delta \mu=f^{n}, \\
& \mu+\beta_{\mu}-P_{1}(\phi)-\Delta \psi=g^{n}, \\
& -\Delta \phi+\psi=0 .
\end{aligned}
$$

with

$$
\int_{\Omega} \phi d x=0, \int_{\Omega} \mu d x=0
$$

We define the inverse laplace operator $v=(-\Delta)^{-1} u$ as follows,

$$
\left\{\begin{array}{l}
-\Delta v=u, \\
\int_{\Omega} v d x=0 .
\end{array}\right.
$$

with the periodic boundary condition or no-flux boundary condition $\left.\partial_{n} v\right|_{\partial \Omega}=0$. Applying $(-\Delta)^{-1}$ to $(3.27)$ and using (3.28)-(3.29), we obtain

$$
\frac{1}{M \delta t}(-\Delta)^{-1} \phi+P_{1}(\phi)-\beta_{\mu}+\Delta^{2} \phi=(-\Delta)^{-1} f^{n}-g^{n}
$$

Let us express the above linear equation (3.32) as $\mathbb{A} \phi=b$. One can verify that for any $\bar{\phi}$ smooth enough such that $\int_{\Omega} \bar{\phi} d x=0$ there holds

$$
(\mathbb{A} \phi, \bar{\phi})=(\phi, \mathbb{A} \bar{\phi}) .
$$

Moreover, it is clear that

$$
\begin{aligned}
(\mathbb{A} \phi, \phi) & =\left(\frac{1}{M \delta t}(-\Delta)^{-1} \phi+P_{1}(\phi)-\beta_{\mu}+\Delta^{2} \phi, \phi\right) \\
& =\frac{1}{M \delta t}\|\phi\|_{H^{-1}}^{2}+\left(P_{1}(\phi), \phi\right)+\|\Delta \phi\|^{2} \geq 0,
\end{aligned}
$$

with "=" if only if $\phi=0$, a fact from Eq. (3.24).

The proof is complete. 
A few remarks are in order.

Remark 3.2. Theorem 3.1 shows that the scheme (3.19)-(3.21) gives a symmetric positive definite linear system for the order parameter $\phi^{n+1}$ when the scheme is further discretized in space. Even solved as a system for the variables $\phi^{n+1}, \mu^{n+1}, \psi^{n+1}$, as is a common practice when the finite element or finite difference methods are used for spatial discretization, the scheme (3.14)-(3.17) still gives a linear system of special properties. We note that, for $M(\phi)=M, \mu^{n+1}, \phi^{n+1}, U^{n+1}, \psi^{n+1}$ are the solutions of

$$
\begin{aligned}
& \phi-\delta t M \Delta \mu=f_{1}, \\
& -\mu+U \phi^{n}+(a-\eta) \phi+\Delta \psi=f_{2}, \\
& \frac{1}{2} U-\phi^{n} \phi=f_{3}, \\
& \psi-\Delta \phi=f_{4} .
\end{aligned}
$$

It can be written in the matrix form as $A \mathbf{x}=b$ where $\mathbf{x}=[\mu, \phi, U, \psi]^{T}, b=\left[f_{1}, f_{2}, f_{3}, f_{4}\right]$. We multiply $\mathbf{x}^{T}=[\mu, \phi, U, \psi]$ to both sides and perform integration by parts. We have

$$
\mathbf{x}^{T} A \mathbf{x}=\frac{1}{2}\|U\|^{2}+(a-\eta)\|\phi\|^{2}+\|\psi\|^{2}+\delta t M\|\nabla \mu\|^{2} \geq 0 .
$$

This implies in particular the uniqueness of the solution to the system (3.35), as the equality sign in Eq. (3.36) yields $\phi=U=\psi=0$ which in turn implies $\mu=0$ in light of the second equation in the system (3.35). Moreover, if one rewrites the second equation in the system (3.35) as

$$
\mu-U \phi^{n}-(a-\eta) \phi-\Delta \psi=-f_{2},
$$

one can verify that the operator matrix $A$ is symmetric. Alternatively, one can obtain a matrix with a positive definite symmetric part (i.e., $\frac{1}{2}\left(A^{T}+A\right)$ ) by reformulating the system (3.35) and restricting the variables $\phi$ and $\mu$ to be of mean zero, see the appendix in [11] for details. Efficient solvers exist for solving such linear systems.

Remark 3.3. In [24,31], a first order convex-splitting scheme is proposed

$$
\begin{aligned}
& \frac{\phi^{n+1}-\phi^{n}}{\delta t}=\nabla \cdot\left(M\left(\phi^{n}\right) \nabla \mu^{n+1}\right), \\
& \mu^{n+1}=\left(\phi^{n+1}\right)^{3}+(a-\eta) \phi^{n+1}+2 a \Delta \phi^{n}+\Delta \psi^{n+1}, \\
& \psi^{n+1}=\Delta \phi^{n+1} .
\end{aligned}
$$

If the Newton iterative method is applied for solving the nonlinear system, at each iteration it would yield the same linear system (3.19)-(3.21) as given by our first order scheme. Hence the cost of solving our scheme is the same as the cost of performing one iteration of Newton method for the nonlinear scheme, provided that the same linear solvers are applied (for instance multi-grid with Gauss-Seidel relaxation). It is clear that our scheme would be much more efficient than the nonlinear convex-splitting schemes. 
Remark 3.4. As we shall show below, the scheme (3.14)-(3.17) is unconditionally energy stable. To the best of the authors knowledge, this is the first linear scheme with energy dissipation law for the PFC model.

Theorem 3.2. The scheme (3.14)-(3.17) admits a unique solution of $\left(\phi^{n+1}, U^{n+1}, \psi^{n+1}\right)$ satisfying the following discrete energy dissipation law,

$$
E_{1 s t}\left(\phi^{n+1}, U^{n+1}, \psi^{n+1}\right) \leq E_{1 s t}\left(\phi^{n}, U^{n}, \psi^{n}\right)-\delta t\left\|\sqrt{M\left(\phi^{n}\right)} \nabla \mu^{n+1}\right\|^{2},
$$

where

$$
E_{1 s t}(\phi, U, \psi)=\frac{1}{4}\|U\|^{2}+\frac{a-\eta}{2}\|\phi\|^{2}-a\|\nabla \phi\|^{2}+\frac{1}{2}\|\psi\|^{2}
$$

Proof. The proof essentially follows the derivation outlined in (3.9)-(3.13). By taking the $L^{2}$ inner product of (3.14) with $-\mu^{n+1}$, we obtain

$$
-\left(\frac{\phi^{n+1}-\phi^{n}}{\delta t}, \mu^{n+1}\right)=\left\|\sqrt{M\left(\phi^{n}\right)} \nabla \mu^{n+1}\right\|^{2},
$$

By taking the $L^{2}$ inner product of (3.15) with $\frac{\phi^{n+1}-\phi^{n}}{\delta t}$, and in view of the following identities

$$
2(a-b, a)=|a|^{2}-|b|^{2}+|a-b|^{2}, \quad 2(a-b, b)=|a|^{2}-|b|^{2}-|a-b|^{2},
$$

we obtain

$$
\begin{aligned}
\left(\mu^{n+1}, \frac{\phi^{n+1}-\phi^{n}}{\delta t}\right)= & \left(U^{n+1} \phi^{n}, \frac{\phi^{n+1}-\phi^{n}}{\delta t}\right)+\frac{1}{\delta t} \frac{a-\eta}{2}\left(\left\|\phi^{n+1}\right\|^{2}-\left\|\phi^{n}\right\|^{2}+\left\|\phi^{n+1}-\phi^{n}\right\|^{2}\right) \\
& -\frac{1}{\delta t} a\left(\left\|\nabla \phi^{n+1}\right\|^{2}-\left\|\nabla \phi^{n}\right\|^{2}-\left\|\nabla\left(\phi^{n+1}-\phi^{n}\right)\right\|^{2}\right) \\
& -\left(\nabla \psi^{n+1}, \nabla \frac{\phi^{n+1}-\phi^{n}}{\delta t}\right) .
\end{aligned}
$$

By taking the $L^{2}$ inner product of (3.16) with $-\frac{1}{2} U^{n+1}$, we obtain

$$
-\frac{1}{\delta t} \frac{1}{4}\left(\left\|U^{n+1}\right\|^{2}-\left\|U^{n}\right\|^{2}+\left\|U^{n+1}-U^{n}\right\|^{2}\right)=-\left(\phi^{n} \frac{\phi^{n+1}-\phi^{n}}{\delta t}, U^{n+1}\right) .
$$

Subtracting the (3.17) at the $n+1$ step and $n$ step, we obtain

$$
\psi^{n+1}-\psi^{n}=\Delta\left(\phi^{n+1}-\phi^{n}\right)
$$

By taking the $L^{2}$ inner product of (3.43) with $-\frac{1}{\delta t} \psi^{n+1}$, we obtain

$$
-\frac{1}{\delta t} \frac{1}{2}\left(\left\|\psi^{n+1}\right\|^{2}-\left\|\psi^{n}\right\|^{2}+\left\|\psi^{n+1}-\psi^{n}\right\|^{2}\right)=\frac{1}{\delta t}\left(\nabla\left(\phi^{n+1}-\phi^{n}\right), \nabla \psi^{n+1}\right) .
$$


Finally, by combinning (3.39)-(3.41)-(3.42)-(3.44), we obtain

$$
\begin{aligned}
& \frac{1}{4}\left(\left\|U^{n+1}\right\|^{2}-\left\|U^{n}\right\|^{2}+\left\|U^{n+1}-U^{n}\right\|^{2}\right) \\
& +\frac{a-\eta}{2}\left(\left\|\phi^{n+1}\right\|^{2}-\left\|\phi^{n}\right\|^{2}+\left\|\phi^{n+1}-\phi^{n}\right\|^{2}\right) \\
& -a\left(\left\|\nabla \phi^{n+1}\right\|^{2}-\left\|\nabla \phi^{n}\right\|^{2}-\left\|\nabla\left(\phi^{n+1}-\phi^{n}\right)\right\|^{2}\right) \\
& +\frac{1}{2}\left(\left\|\psi^{n+1}\right\|^{2}-\left\|\psi^{n}\right\|^{2}+\left\|\psi^{n+1}-\psi^{n}\right\|^{2}\right)=-\delta t\left\|\sqrt{M\left(\phi^{n}\right)} \nabla \mu^{n+1}\right\|^{2},
\end{aligned}
$$

which implies the result (3.37) after we drop some positive terms.

3.2. The second-order schemes. Now we construct two classes of second order schemes. One is based on the BDF2 time discretization, and the other is based on the Crank-Nicolson method.

3.2.1. The BDF2 scheme. Given the initial conditions $\phi^{0}, U^{0}$, we first compute $\phi^{1}, U^{1}$ by the first order scheme (3.14)-(3.17). Having computed $\phi^{n-1}, U^{n-1}$ and $\phi^{n}, U^{n}$ for $n \geq 1$, we compute $\phi^{n+1}, U^{n+1}$ by the following second order BDF2 scheme.

$$
\begin{aligned}
& \frac{3 \phi^{n+1}-4 \phi^{n}+\phi^{n-1}}{2 \delta t}=\nabla \cdot\left(M\left(2 \phi^{n}-\phi^{n-1}\right) \nabla \mu^{n+1}\right), \\
& \mu^{n+1}=U^{n+1}\left(2 \phi^{n}-\phi^{n-1}\right)+(a-\eta) \phi^{n+1}+2 a \Delta\left(2 \phi^{n}-\phi^{n-1}\right)+\Delta \psi^{n+1}, \\
& \frac{3 U^{n+1}-4 U^{n}+U^{n-1}}{2 \delta t}=2\left(2 \phi^{n}-\phi^{n-1}\right) \frac{3 \phi^{n+1}-4 \phi^{n}+\phi^{n-1}}{2 \delta t}, \\
& \psi^{n+1}=\Delta \phi^{n+1} .
\end{aligned}
$$

Remark 3.5. As for the first order scheme (3.14)-(3.17), we can easily show the(3.46)-(3.49) is a positive definite matrix system. We rewrite (3.48) as

$$
U^{n+1}=\alpha_{2}+\beta_{2} \phi^{n+1},
$$

where $\alpha_{2}=\frac{2}{3}\left(2 \phi^{n}-\phi^{n-1}\right)\left(-4 \phi^{n}+\phi^{n-1}\right)+\frac{1}{3}\left(4 U^{n}-U^{n-1}\right), \beta_{2}=2\left(2 \phi^{n}-\phi^{n-1}\right)$. In turn, $(3.47)$ can be rewritten as

$$
\mu^{n+1}=P_{2}\left(\phi^{n+1}\right)+\Delta \psi^{n+1}+\tilde{\alpha}_{2},
$$

where

$$
\left\{\begin{array}{l}
P_{2}\left(\phi^{n+1}\right)=\tilde{\alpha}_{2} \phi^{n+1}+(a-\eta) \phi^{n+1}, \\
\tilde{\alpha}_{2}=\alpha_{2}\left(2 \phi^{n}-\phi^{n-1}\right)+2 a \Delta\left(2 \phi^{n}-\phi^{n-1}\right) .
\end{array}\right.
$$

Moreover, similar to the first-order scheme, when the mobility parameter $M(\phi)$ is a positive constant $M$, we can show the linear system is symmetric positive definite for $\phi^{n+1}$.

Theorem 3.3. The scheme (3.46)-(3.49) is unconditionally stable in the sense that it satisfies the following energy inequality.

$$
E_{b d f 2}^{n+1, n} \leq E_{b d f 2}^{n, n-1}-\delta t\left\|\sqrt{M\left(2 \phi^{n}-\phi^{n-1}\right)} \nabla \mu^{n+1}\right\|^{2},
$$


where

$$
\begin{aligned}
E_{b d f 2}^{n+1, n}= & \frac{1}{4}\left(\frac{\left\|U^{n+1}\right\|^{2}+\left\|2 U^{n+1}-U^{n}\right\|^{2}}{2}\right)+\frac{a-\eta}{2}\left(\frac{\left\|\phi^{n+1}\right\|^{2}+\left\|2 \phi^{n+1}-\phi^{n}\right\|^{2}}{2}\right) \\
& -a\left(\frac{\left\|\nabla \phi^{n+1}\right\|^{2}+\left\|\nabla\left(2 \phi^{n+1}-\phi^{n}\right)\right\|^{2}}{2}\right)+\left(\frac{\left\|\psi^{n+1}\right\|^{2}+\left\|2 \psi^{n+1}-\psi^{n}\right\|^{2}}{2}\right) \\
& +a\left\|\nabla\left(\phi^{n+1}-\phi^{n}\right)\right\|^{2} .
\end{aligned}
$$

Proof. By taking the $L^{2}$ inner product of (3.46) with $-\mu^{n+1}$, we obtain

$$
-\left(\frac{3 \phi^{n+1}-4 \phi^{n}+\phi^{n-1}}{2 \delta t}, \mu^{n+1}\right)=\left\|\sqrt{M\left(2 \phi^{n}-\phi^{n-1}\right)} \nabla \mu^{n+1}\right\|^{2},
$$

By taking the $L^{2}$ inner product of (3.47) with $\frac{3 \phi^{n+1}-4 \phi^{n}+\phi^{n-1}}{2 \delta t}$, and applying the following identities

$$
\begin{aligned}
2(3 a-4 b+c, a) & =|a|^{2}-|b|^{2}+|2 a-b|^{2}-|2 b-c|^{2}+|a-2 b+c|^{2}, \\
2(3 a-4 b+c, 2 b-c) & =\left(|a|^{2}+|2 a-b|^{2}-2|a-b|^{2}\right)-\left(|b|^{2}+|2 b-c|^{2}-2|b-c|^{2}\right) \\
& -3|a-2 b+c|^{2},
\end{aligned}
$$

we obtain

$$
\begin{aligned}
\left(\mu^{n+1}, \frac{3 \phi^{n+1}-4 \phi^{n}+\phi^{n-1}}{2 \delta t}\right) & =\left(U^{n+1}\left(2 \phi^{n}-\phi^{n-1}\right), \frac{3 \phi^{n+1}-4 \phi^{n}+\phi^{n-1}}{2 \delta t}\right) \\
& +\frac{1}{2 \delta t} \frac{a-\eta}{2}\left(\left\|\phi^{n+1}\right\|^{2}+\left\|2 \phi^{n+1}-\phi^{n}\right\|^{2}\right) \\
& -\frac{1}{2 \delta t} \frac{a-\eta}{2}\left(\left\|\phi^{n}\right\|^{2}+\left\|2 \phi^{n}-\phi^{n-1}\right\|^{2}\right)+\frac{1}{2 \delta t} \frac{a-\eta}{2}\left\|\phi^{n+1}-2 \phi^{n}+\phi^{n-1}\right\|^{2} \\
& -\frac{1}{2 \delta t} a\left(\left\|\nabla \phi^{n+1}\right\|^{2}+\left\|\nabla\left(2 \phi^{n+1}-\phi^{n}\right)\right\|^{2}-2\left\|\nabla\left(\phi^{n+1}-\phi^{n}\right)\right\|^{2}\right) \\
& +\frac{1}{2 \delta t} a\left(\left\|\nabla \phi^{n}\right\|^{2}+\left\|\nabla\left(2 \phi^{n}-\phi^{n-1}\right)\right\|^{2}-2\left\|\nabla\left(\phi^{n}-\phi^{n-1}\right)\right\|^{2}\right) \\
& +\frac{3 a}{2 \delta t}\left\|\nabla\left(\phi^{n+1}-2 \phi^{n}+\phi^{n-1}\right)\right\|^{2} \\
& -\left(\nabla \psi^{n+1}, \nabla \frac{3 \phi^{n+1}-4 \phi^{n}+\phi^{n-1}}{2 \delta t}\right) .
\end{aligned}
$$

By taking the $L^{2}$ inner product of (3.48) with $-\frac{1}{2} U^{n+1}$, we obtain

$$
\begin{aligned}
& -\frac{1}{4 \delta t} \frac{1}{2}\left(\left\|U^{n+1}\right\|^{2}-\left\|U^{n}\right\|^{2}+\left\|2 U^{n+1}-U^{n}\right\|^{2}-\left\|2 U^{n}-U^{n-1}\right\|^{2}\right. \\
& \left.\quad+\left\|U^{n+1}-2 U^{n}+U^{n-1}\right\|^{2}\right)=-\left(\left(2 \phi^{n}-\phi^{n-1}\right) \frac{3 \phi^{n+1}-4 \phi^{n}+\phi^{n-1}}{2 \delta t}, U^{n+1}\right) .
\end{aligned}
$$

From (3.49), we have

$$
3 \psi^{n+1}-4 \psi^{n}+\psi^{n-1}=\Delta\left(3 \phi^{n+1}-4 \phi^{n}+\phi^{n-1}\right) .
$$


By taking the $L^{2}$ inner product of (3.58) with $-\frac{1}{2 \delta t} \psi^{n+1}$, we obtain

$$
\begin{gathered}
-\frac{1}{4 \delta t}\left(\left\|\psi^{n+1}\right\|^{2}-\left\|\psi^{n}\right\|^{2}+\left\|2 \psi^{n+1}-\psi^{n}\right\|^{2}-\left\|2 \psi^{n}-\psi^{n-1}\right\|^{2}+\left\|\psi^{n+1}-2 \psi^{n}+\psi^{n-1}\right\|^{2}\right) . \\
=\frac{1}{2 \delta t}\left(\nabla\left(3 \phi^{n+1}-4 \phi^{n}+\phi^{n-1}\right), \nabla \psi^{n+1}\right) .
\end{gathered}
$$

Finally, by combining (3.55)-(3.56)-(3.57)-(3.59), we obtain

$$
\begin{aligned}
& \frac{1}{4}\left(\frac{\left\|U^{n+1}\right\|^{2}+\left\|2 U^{n+1}-U^{n}\right\|^{2}}{2}\right)-\frac{1}{4}\left(\frac{\left\|U^{n}\right\|^{2}+\left\|2 U^{n}-U^{n-1}\right\|^{2}}{2}\right) \\
& +\frac{a-\eta}{2}\left(\frac{\left\|\phi^{n+1}\right\|^{2}+\left\|2 \phi^{n+1}-\phi^{n}\right\|^{2}}{2}\right)-\frac{a-\eta}{2}\left(\frac{\left\|\phi^{n}\right\|^{2}+\left\|2 \phi^{n}-\phi^{n-1}\right\|^{2}}{2}\right) \\
& -a\left(\frac{\left\|\nabla \phi^{n+1}\right\|^{2}+\left\|\nabla\left(2 \phi^{n+1}-\phi^{n}\right)\right\|^{2}-2\left\|\nabla\left(\phi^{n+1}-\phi^{n}\right)\right\|^{2}}{2}\right) \\
& +a\left(\frac{\left\|\nabla \phi^{n}\right\|^{2}+\left\|\nabla\left(2 \phi^{n}-\phi^{n-1}\right)\right\|^{2}-2\left\|\nabla\left(\phi^{n}-\phi^{n-1}\right)\right\|^{2}}{2}\right) \\
& +\left(\frac{\left\|\psi^{n+1}\right\|^{2}+\left\|2 \psi^{n+1}-\psi^{n}\right\|^{2}}{2}\right)-\left(\frac{\left\|\psi^{n}\right\|^{2}+\left\|2 \psi^{n}-\psi^{n-1}\right\|^{2}}{2}\right) \\
& +\frac{a-\eta}{4}\left\|\phi^{n+1}-2 \phi^{n}+\phi^{n-1}\right\|^{2}+\frac{3 a}{2}\left\|\nabla\left(\phi^{n+1}-2 \phi^{n}+\phi^{n-1}\right)\right\|^{2}+\frac{1}{4}\left\|\psi^{n+1}-2 \psi^{n}+\psi^{n-1}\right\| \\
& +\frac{1}{2}\left\|U^{n+1}-2 U^{n}+U^{n-1}\right\|^{2}=-\delta t\left\|\sqrt{M\left(2 \phi^{n}-\phi^{n-1}\right)} \nabla \mu^{n+1}\right\|^{2},
\end{aligned}
$$

which implies the result (3.53) after we drop some positive terms.

Remark 3.6. Heuristically, the discrete energy law (3.53) is a second order approximation of the continuous energy law (2.5), as $\left(E_{b d f 2}^{n+1, n}-E_{b d f 2}^{n, n-1}\right) / \delta t$ is a second order approximation of $\frac{d}{d t} E(\phi, U, \psi)$ at time $t=t^{n+1}$, cf. Eq. (3.2). For instance, one can write

$$
\begin{aligned}
& \left(\frac{\left\|U^{n+1}\right\|^{2}+\left\|2 U^{n+1}-U^{n}\right\|^{2}}{2 \delta t}\right)-\left(\frac{\left\|U^{n}\right\|^{2}+\left\|2 U^{n}-U^{n-1}\right\|^{2}}{2 \delta t}\right) \\
& \cong\left(\frac{\left\|U^{n+2}\right\|^{2}-\left\|U^{n}\right\|^{2}}{2 \delta t}\right)+O\left(\delta t^{2}\right) \cong \frac{d}{d t}\left\|U\left(t^{n+1}\right)\right\|^{2}+O\left(\delta t^{2}\right) .
\end{aligned}
$$

and

and

$$
\left(\frac{\left\|\nabla \phi^{n+1}-\nabla \phi^{n}\right\|^{2}-\left\|\nabla \phi^{n}-\nabla \phi^{n-1}\right\|^{2}}{\delta t}\right) \cong O\left(\delta t^{2}\right)
$$

3.2.2. The Crank-Nicolson scheme. Given the initial conditions $\phi^{0}, U^{0}$, we compute $\phi^{1}, U^{1}$ by the first order scheme. Having computed $\phi^{n-1}, U^{n-1}$ and $\phi^{n}, U^{n}$ for $n \geq 1$, we compute $\phi^{n+1}, U^{n+1}$ by the following second order Crank-Nicolson scheme. 


$$
\begin{aligned}
& \frac{\phi^{n+1}-\phi^{n}}{\delta t}=\nabla \cdot\left(M\left(\frac{3}{2} \phi^{n}-\frac{1}{2} \phi^{n-1}\right) \nabla \mu^{n+\frac{1}{2}}\right), \\
& \mu^{n+\frac{1}{2}}=\frac{U^{n+1}+U^{n}}{2}\left(\frac{3}{2} \phi^{n}-\frac{1}{2} \phi^{n-1}\right)+(a-\eta) \frac{\phi^{n+1}+\phi^{n}}{2} \\
& +2 a \Delta\left(\frac{3}{2} \phi^{n}-\frac{1}{2} \phi^{n-1}\right)+\Delta\left(\frac{\psi^{n+1}+\psi^{n}}{2}\right), \\
& \frac{U^{n+1}-U^{n}}{\delta t}=2\left(\frac{3}{2} \phi^{n}-\frac{1}{2} \phi^{n-1}\right) \frac{\phi^{n+1}-\phi^{n}}{\delta t}, \\
& \psi^{n+1}=\Delta \phi^{n+1} \text {. }
\end{aligned}
$$

Remark 3.7. We can rewrite (3.63) as

$$
U^{n+1}=\alpha_{3}+\beta_{3} \phi^{n+1}
$$

where $\alpha_{3}=-\left(3 \phi^{n}-\phi^{n-1}\right) \phi^{n}+U^{n}, \beta_{3}=\left(3 \phi^{n}-\phi^{n-1}\right)$. In turn, (3.62) can be rewritten as

$$
\mu^{n+\frac{1}{2}}=P_{3}\left(\phi^{n+1}\right)+\frac{1}{2} \Delta \psi^{n+1}+\tilde{\alpha}_{3},
$$

where

$$
\left\{\begin{array}{l}
P_{3}\left(\phi^{n+1}\right)=\frac{3}{4} \beta_{3}^{2} \phi^{n+1}+\frac{a-\eta}{2} \phi^{n+1} \\
\tilde{\alpha}_{3}=\frac{\alpha_{3}+U^{n}}{2}\left(\frac{3}{2} \phi^{n}-\frac{1}{2} \phi^{n-1}\right)+\frac{a-\eta}{2} \phi^{n}+2 a \Delta\left(\frac{3}{2} \phi^{n}-\frac{1}{2} \phi^{n-1}\right)+\frac{1}{2} \Delta \psi^{n} .
\end{array}\right.
$$

Moreover, similar to the first-order scheme and the second order BDF2 scheme, when the mobility parameter $M(\phi)$ is a positive constant $M$, we can show the linear system is symmetric postive definite for $\phi^{n+1}$.

Theorem 3.4. The scheme (3.61)-(3.64) is unconditionally stable, and admits a unique solution satisfying the following discrete energy dissipation law.

$$
E_{c n 2}^{n+1, n} \leq E_{c n 2}^{n, n-1}-\delta t\left\|\sqrt{M\left(\frac{3}{2} \phi^{n}-\frac{1}{2} \phi^{n-1}\right)} \nabla \mu^{n+\frac{1}{2}}\right\|^{2},
$$

where

$$
E_{c n 2}^{n+1, n}=\frac{1}{4}\left\|U^{n+1}\right\|^{2}+\frac{a-\eta}{2}\left\|\phi^{n+1}\right\|^{2}-a\left\|\nabla \phi^{n+1}\right\|^{2}+\frac{\left\|\psi^{n+1}\right\|^{2}}{2}+\frac{a}{2}\left\|\nabla\left(\phi^{n+1}-\phi^{n}\right)\right\|^{2} .
$$

Proof. By taking the $L^{2}$ inner product of (3.61) with $-\mu^{n+\frac{1}{2}}$, we obtain

$$
-\left(\frac{\phi^{n+1}-\phi^{n}}{\delta t}, \mu^{n+\frac{1}{2}}\right)=\left\|\sqrt{M\left(\frac{3}{2} \phi^{n}-\frac{1}{2} \phi^{n-1}\right)} \nabla \mu^{n+\frac{1}{2}}\right\|^{2} .
$$

By taking the $L^{2}$ inner product of (3.62) with $\frac{\phi^{n+1}-\phi^{n}}{\delta t}$, and applying the following identity

$$
\frac{1}{2}(3 b-c, a-b)=\frac{1}{2}\left(|a|^{2}-|b|^{2}\right)-\frac{1}{4}\left(|a-b|^{2}-|b-c|^{2}+|a-2 b+c|^{2}\right),
$$


we obtain

$$
\begin{aligned}
\left(\mu^{n+\frac{1}{2}}, \frac{\phi^{n+1}-\phi^{n}}{\delta t}\right) & =\left(\frac{1}{2}\left(U^{n+1}+U^{n}\right)\left(\frac{3}{2} \phi^{n}-\frac{1}{2} \phi^{n-1}\right), \frac{\phi^{n+1}-\phi^{n}}{\delta t}\right) \\
& +\frac{1}{\delta t} \frac{(a-\eta)}{2}\left(\left\|\phi^{n+1}\right\|^{2}-\left\|\phi^{n}\right\|^{2}\right) \\
& -\frac{1}{\delta t} a\left(\left\|\nabla \phi^{n+1}\right\|^{2}-\frac{1}{2}\left\|\nabla\left(\phi^{n+1}-\phi^{n}\right)\right\|^{2}\right) \\
& +\frac{1}{\delta t} a\left(\left\|\nabla \phi^{n}\right\|^{2}-\frac{1}{2}\left\|\nabla\left(\phi^{n}-\phi^{n-1}\right)\right\|^{2}\right) \\
& +\frac{a}{2 \delta t}\left\|\nabla\left(\phi^{n+1}-2 \phi^{n}+\phi^{n-1}\right)\right\|^{2} \\
& -\left(\nabla \frac{\psi^{n+1}+\psi^{n}}{2}, \nabla \frac{\phi^{n+1}-\phi^{n}}{\delta t}\right) .
\end{aligned}
$$

By taking the $L^{2}$ inner product of (3.63) with $-\frac{1}{4}\left(U^{n+1}+U^{n}\right)$, we obtain

$$
-\frac{1}{4 \delta t}\left(\left\|U^{n+1}\right\|^{2}-\left\|U^{n}\right\|^{2}\right)=-\left(\left(\frac{3}{2} \phi^{n}-\frac{1}{2} \phi^{n-1}\right) \frac{\phi^{n+1}-\phi^{n}}{\delta t}, \frac{U^{n+1}+U^{n}}{2}\right) .
$$

From (3.64), we have

$$
\frac{\psi^{n+1}-\psi^{n}}{2}=\Delta \frac{\phi^{n+1}-\phi^{n}}{2} .
$$

By taking the $L^{2}$ inner product of (3.73) with $-\frac{\psi^{n+1}+\psi^{n}}{\delta t}$, we obtain

$$
-\frac{1}{\delta t}\left(\frac{1}{2}\left\|\psi^{n+1}\right\|^{2}-\frac{1}{2}\left\|\psi^{n}\right\|^{2}\right)=\frac{1}{\delta t}\left(\nabla \frac{\phi^{n+1}-\phi^{n}}{2}, \nabla\left(\psi^{n+1}+\psi^{n}\right)\right) .
$$

Finally, by combining (3.69)-(3.71)-(3.72)-(3.74), we obtain

$$
\begin{aligned}
& \frac{1}{4}\left(\left\|U^{n+1}\right\|^{2}-\left\|U^{n}\right\|^{2}\right)+\frac{a-\eta}{2}\left(\left\|\phi^{n+1}\right\|^{2}-\left\|\phi^{n}\right\|^{2}\right) \\
& -a\left(\left\|\nabla \phi^{n+1}\right\|^{2}-\frac{1}{2}\left\|\nabla \phi^{n+1}-\nabla \phi^{n}\right\|^{2}\right)+a\left(\left\|\nabla \phi^{n}\right\|^{2}-\frac{1}{2}\left\|\nabla \phi^{n}-\nabla \phi^{n-1}\right\|^{2}\right) \\
& +\frac{1}{2}\left(\left\|\psi^{n+1}\right\|^{2}-\left\|\psi^{n}\right\|^{2}\right)+\frac{a}{2}\left\|\nabla\left(\phi^{n+1}-2 \phi^{n}+\phi^{n-1}\right)\right\|^{2} \\
& =-\delta t\left\|\sqrt{M\left(\frac{3}{2} \phi^{n}-\frac{1}{2} \phi^{n-1}\right)} \nabla \mu^{n+\frac{1}{2}}\right\|^{2} .
\end{aligned}
$$

The inequality (3.68) follows from (3.75) after we drop some positive terms.

Remark 3.8. One can formally verify that the energy law (3.68) is a second order approximation of the continuous energy law (2.5) at time level $t^{n+\frac{1}{2}}$.

Remark 3.9. It is clear from the proof of Theorems 3.2, 3.3 and 3.4 that the same energy stability holds or the fully discrete schemes if the schemes (3.14)-(3.17), (3.46)-(3.49), (3.61)-(3.64) are further discretized in space, for instance, by finite difference, finite element or spectral methods.

Remark 3.10. The local truncation error of the schemes (3.14)-(3.17), (3.46)-(3.49) and (3.61)(3.64) can be readily derived via Taylor series expansion assuming that the solutions and the 
mobility functions have enough regularity. We omit the details here for brevity. The complete error analysis of the schemes will be pursued in a separate article.

\section{Numerical Simulations}

In this section, we present some numerical results to validate the efficiency and accuracy of the proposed schemes. We choose the periodic boundary conditions and use the Fourier-spectral method to discretize the variables in space. We note that periodic boundary conditions are relevant in the study of PFC, cf. [24,31].

4.1. Accuracy test. We test the convergence rates of the three proposed schemes, the firstorder scheme (3.14)-(3.17), denoted by LS1, the second-order BDF2 scheme (3.46)-(3.49), denoted by BDF2; and the second order Crank-Nicolson scheme (3.61)-(3.64), denoted by CN2. The computational domain is set to be $\Omega=[0,128]^{2}$ and the order parameters are $\eta=0.025, a=$ $1, M=1$.

\begin{tabular}{c|c|c|c|c|c|c}
\hline$\delta t$ & LS1 & Order & CN2 & Order & BDF2 & Order \\
\hline 1 & 1.28 & & $1.32 \mathrm{E}(-1)$ & & $6.01 \mathrm{E}(-1)$ & \\
\hline 0.5 & $1.4 \mathrm{E}(-1)$ & 3.20 & $3.22 \mathrm{E}(-2)$ & 2.04 & $5.39 \mathrm{E}(-2)$ & 3.48 \\
\hline 0.1 & $2.18 \mathrm{E}(-2)$ & 1.16 & $1.29 \mathrm{E}(-3)$ & 2.00 & $1.85 \mathrm{E}(-3)$ & 2.10 \\
\hline 0.05 & $1.09 \mathrm{E}(-2)$ & 1.00 & $3.23 \mathrm{E}(-4)$ & 2.00 & $4.5 \mathrm{E}(-4)$ & 2.04 \\
\hline 0.01 & $2.19 \mathrm{E}(-3)$ & 1.00 & $1.30 \mathrm{E}(-5)$ & 2.00 & $1.77 \mathrm{E}(-5)$ & 2.01 \\
\hline 0.005 & $1.09 \mathrm{E}(-3)$ & 1.00 & $3.21 \mathrm{E}(-6)$ & 2.02 & $4.49 \mathrm{E}(-6)$ & 1.98 \\
\hline 0.001 & $2.19 \mathrm{E}(-4)$ & 1.00 & $1.27 \mathrm{E}(-7)$ & 2.01 & $1.77 \mathrm{E}(-7)$ & 2.01 \\
\hline
\end{tabular}

TABLE 4.1. The numerical errors at $t=20$ for the phase variable $\phi$ that are computed by LS1, CN2 and BDF2 schemes using different temporal resolutions. The exact solution is given in (4.1) with order parameters $\eta=0.025, a=1, M=1$ and $257^{2}$ Fourier modes for space.

We perform two numerical simulations to test the accuracy. In the first example, we choose the suitable forcing functions such that the exact solution is given by

$$
\phi(t, x, y)=\sin \left(\frac{2 \pi}{64} x\right) \cos \left(\frac{2 \pi}{64} y\right) \cos (t) .
$$

We use 257 Fourier modes so the spatial discretization errors are negligible compared with the time discretization error. In Table 4.1, we list the $L^{2}$ errors of the phase variable between the numerical solution and the exact solution at $t=20$ with different time step sizes. 


\begin{tabular}{c|c|c|c|c|c|c}
\hline$\delta t$ & LS1 & Order & CN2 & Order & BDF2 & Order \\
\hline 1 & $2.6 \mathrm{E}(-3)$ & & $2.56 \mathrm{E}(-4)$ & & $5.97 \mathrm{E}(-4)$ & \\
\hline 0.5 & $1.3 \mathrm{E}(-3)$ & 1.00 & $6.35 \mathrm{E}(-5)$ & 2.01 & $1.33 \mathrm{E}(-4)$ & 2.17 \\
\hline 0.1 & $2.52 \mathrm{E}(-4)$ & 1.02 & $2.64 \mathrm{E}(-6)$ & 1.98 & $5.59 \mathrm{E}(-6)$ & 1.97 \\
\hline 0.05 & $1.25 \mathrm{E}(-4)$ & 1.02 & $6.66 \mathrm{E}(-7)$ & 1.99 & $1.41 \mathrm{E}(-6)$ & 1.98 \\
\hline 0.01 & $2.29 \mathrm{E}(-5)$ & 1.05 & $2.65 \mathrm{E}(-8)$ & 2.00 & $5.64 \mathrm{E}(-8)$ & 1.99 \\
\hline 0.005 & $1.01 \mathrm{E}(-5)$ & 1.18 & $6.45 \mathrm{E}(-9)$ & 2.04 & $1.37 \mathrm{E}(-8)$ & 2.04 \\
\hline
\end{tabular}

TABLE 4.2. The numerical errors at $t=20$ for the phase variable $\phi$ that are computed by LS1, CN2 and BDF2 schemes using different temporal resolutions. The numerical results using $\delta t=0.001$ is taken as the exact solution to calculate the $L^{2}$ errors with order parameters $\eta=0.025, a=1, M=1$ and $257^{2}$ fourier modes for space.

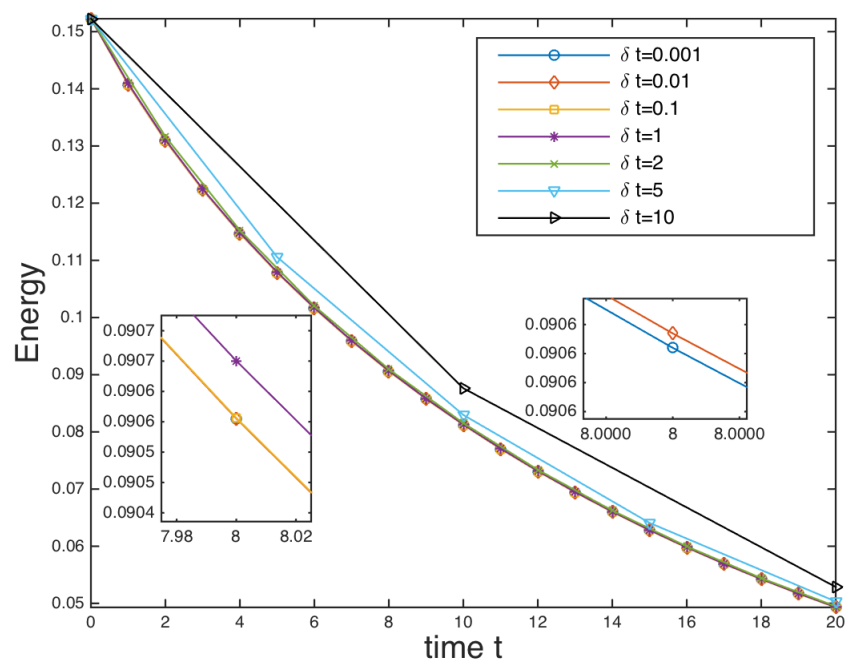

Figure 4.1. Time evolution of the energy functional for five different time steps of $\delta t=0.001,0.01,0.1,1,5,10$. The small inset figure shows the small differences in the energy evolution for the considered time steps of $\delta t=0.001,0.01,0.1$ and 1.

In the second example, we perform the mesh refinement test with the following initial condition

$$
\phi(0, x, y)=\sin \left(\frac{2 \pi}{64} x\right) \cos \left(\frac{2 \pi}{64} y\right) \text {. }
$$



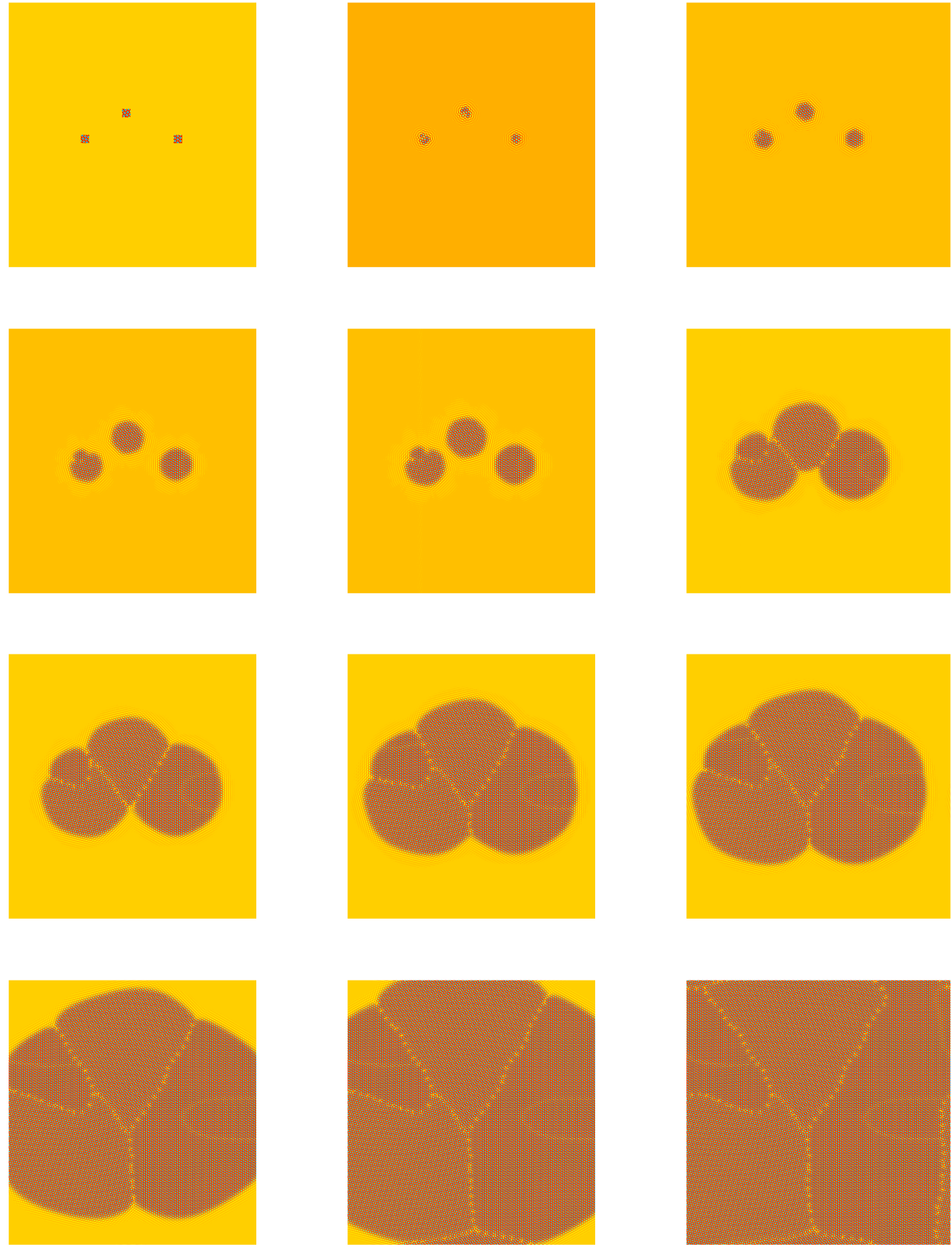

FiguRE 4.2. The dynamical behaviors of the crystal growth in a supercooled liquid. Snapshots of the numerical approximation of $\phi$ to the atomistic density field at $t=0,40,100,160,200,300,400,500,600,800,1000,1500$. The computational domain is $[0,800]^{2}$. The order parameters of are $\eta=0.25, M=1, a=1.513^{2}$ Fourier modes are used to discretize the space. The time step is $\delta t=0.01$. 


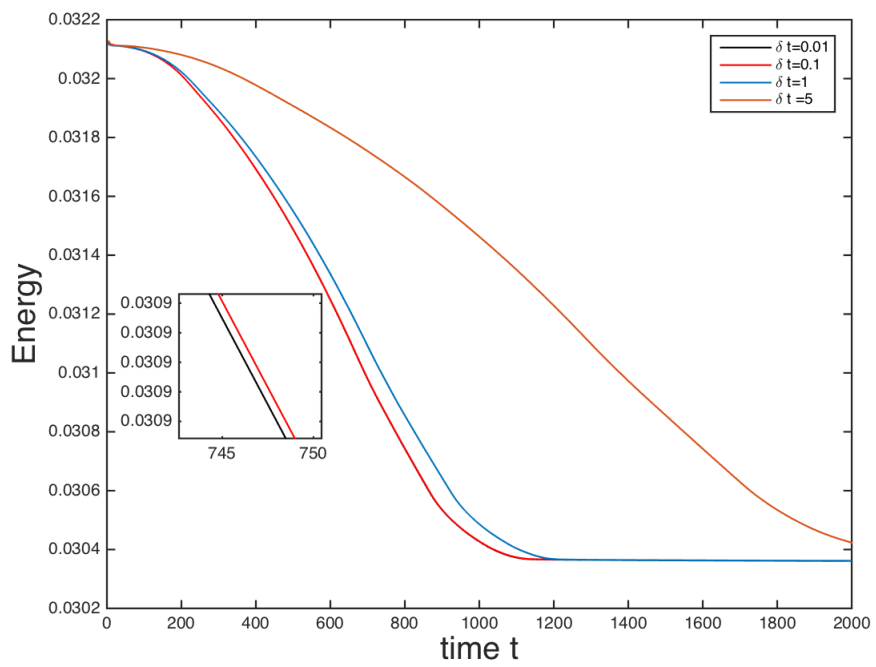

Figure 4.3. Time evolution of the free energy functional for four different time steps of $\delta t=0.01,0.1,1,5$. The energy curves show the decays for all time steps, which confirms that our algorithm is unconditionally stable. The small inset figure shows the small differences in the energy evolution for the considered time steps of $\delta t=0.01$ and $\delta t=0.1$.

All parameters are the same as the first example and we choose the $\delta t=0.001$ as the exact solution. In Table 4.2, we list the $L^{2}$ errors of the phase variable between the numerical solution and the exact solution at $t=20$ with different time step sizes.

From Table 4.1 and 4.2, we can observe that

- all three schemes present the good convergence rate that asymptotically (at least) match their corresponding orders for time, thus good approximations to the exact solution.

- the BDF2 and CN2 give much better accuracy than the LS1. For instances, when $\delta t=1$, the $L^{2}$ error of LS1 is as big as $o(1)$ that is actually very away from the exact solution, while the $L^{2}$ errors from CN2 and BDF2 can reach $o\left(10^{-1}\right)$. When $\delta t=0.001$, the $L^{2}$ error of LS1 is just $o\left(10^{-4}\right)$, and the error of BDF2 and CN2 can reach $o\left(10^{-7}\right)$.

- the CN2 performs slightly better than BDF2 when using the same time step. For example, when $\delta t=0.001$, the $L^{2}$ error of CN2 is just $1 / 2$ of the error of BDF2.

In Figure. 4.1, we plot the time evolution of the energy functional with different time step size of $\delta t=0.001,0.01,0.1,1,2,5,10$ using the CN2 scheme. Up to $\delta t=2$, the numerical solution matches well with the exact solution of $\delta t=0.001$. When we use very large time step as $\delta t=5,10$, we see that the obtained energy curve is considerably away from the exact solution.

4.2. Crystal growth in a supercooled liquid in 2D and 3D. We simulate the growth of a polycrystal in a supercooled liquid. For 2D space, initially, three crystallites with different 

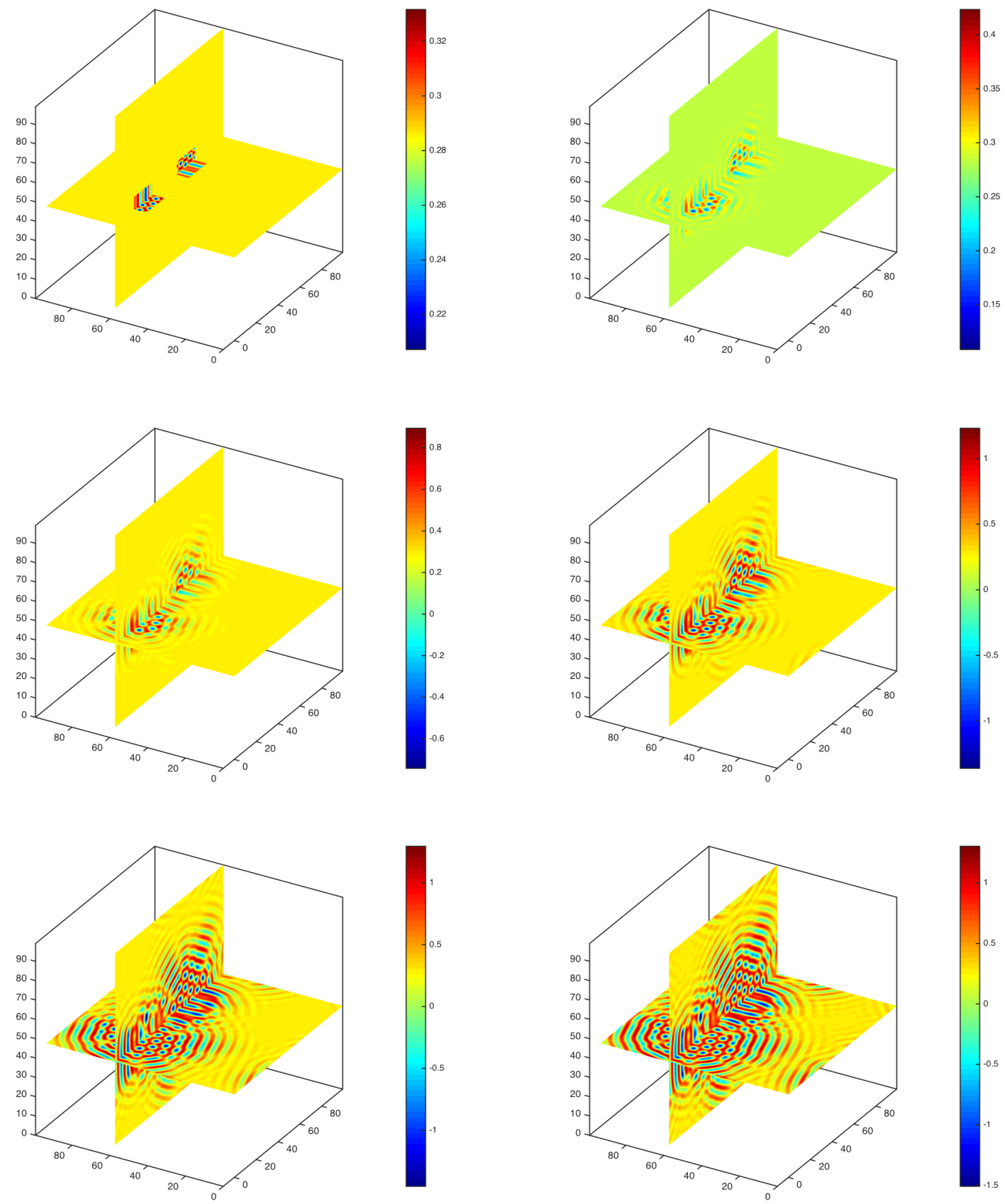

FiguRE 4.4. The 3D dynamical behaviors of the crystal growth in a supercooled liquid. Snapshots of the numerical approximation of $\phi$ to the atomistic density field at $t=0,2.5,5,7,9,10$. The computational domain is $[0,100]^{2}$. The order parameters of are $\eta=1, M=1, a=1.128^{3}$ Fourier modes are used to discretize the 3D domain. The time step is $\delta t=0.01$. 

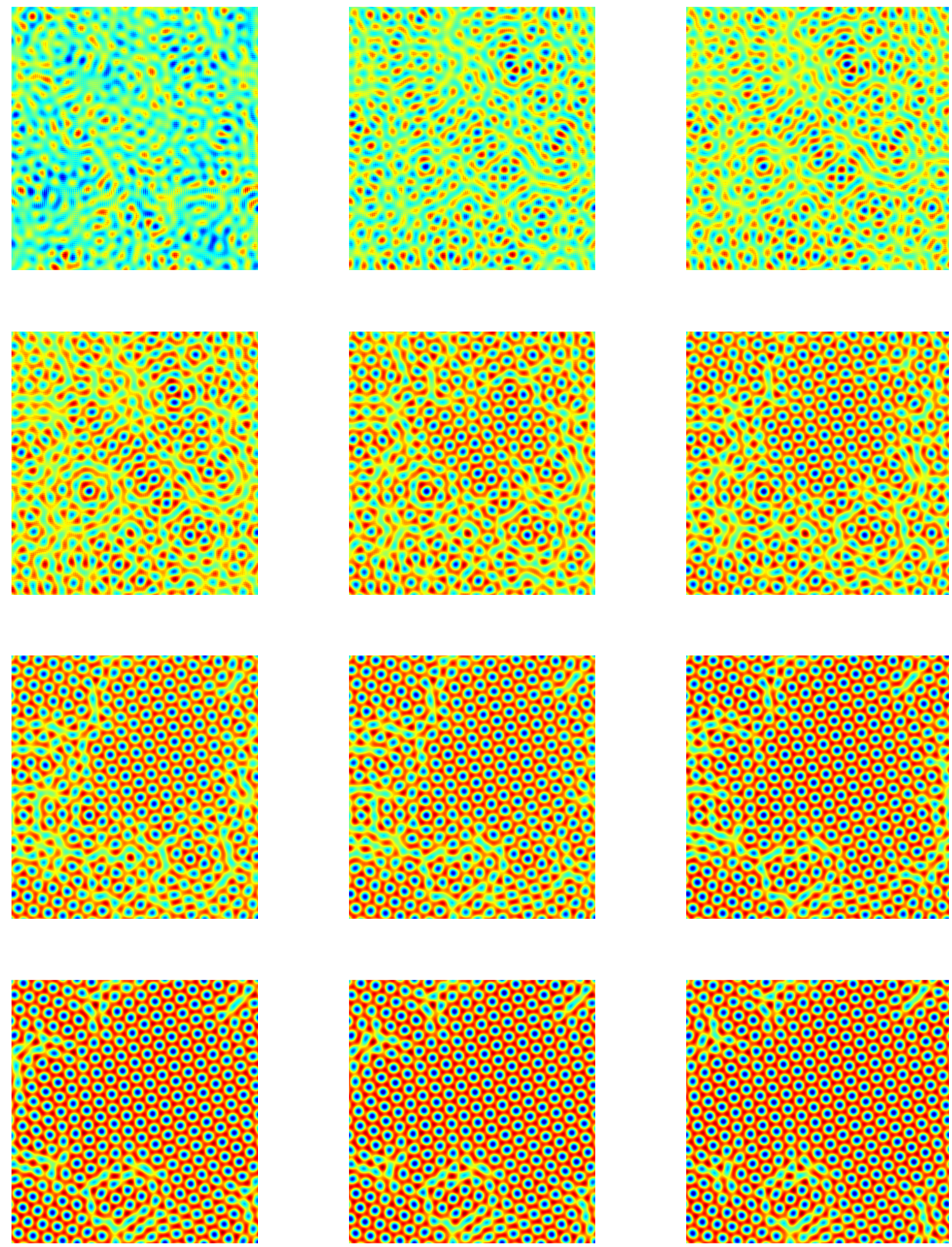

FiguRE 4.5. The dynamical behaviors of the phase separation behavior. Snapshots of the numerical approximation of $\phi$ are taken at $t=10,100,200,300,400$, $500,600,700,800,1000,1100,1200$. The computational domain is $[0,128]^{2}$. The order parameters of are $\eta=0.025, M=1, a=1.257^{2}$ Fourier modes are used to discretize the space. The time step is $\delta t=0.001$. 

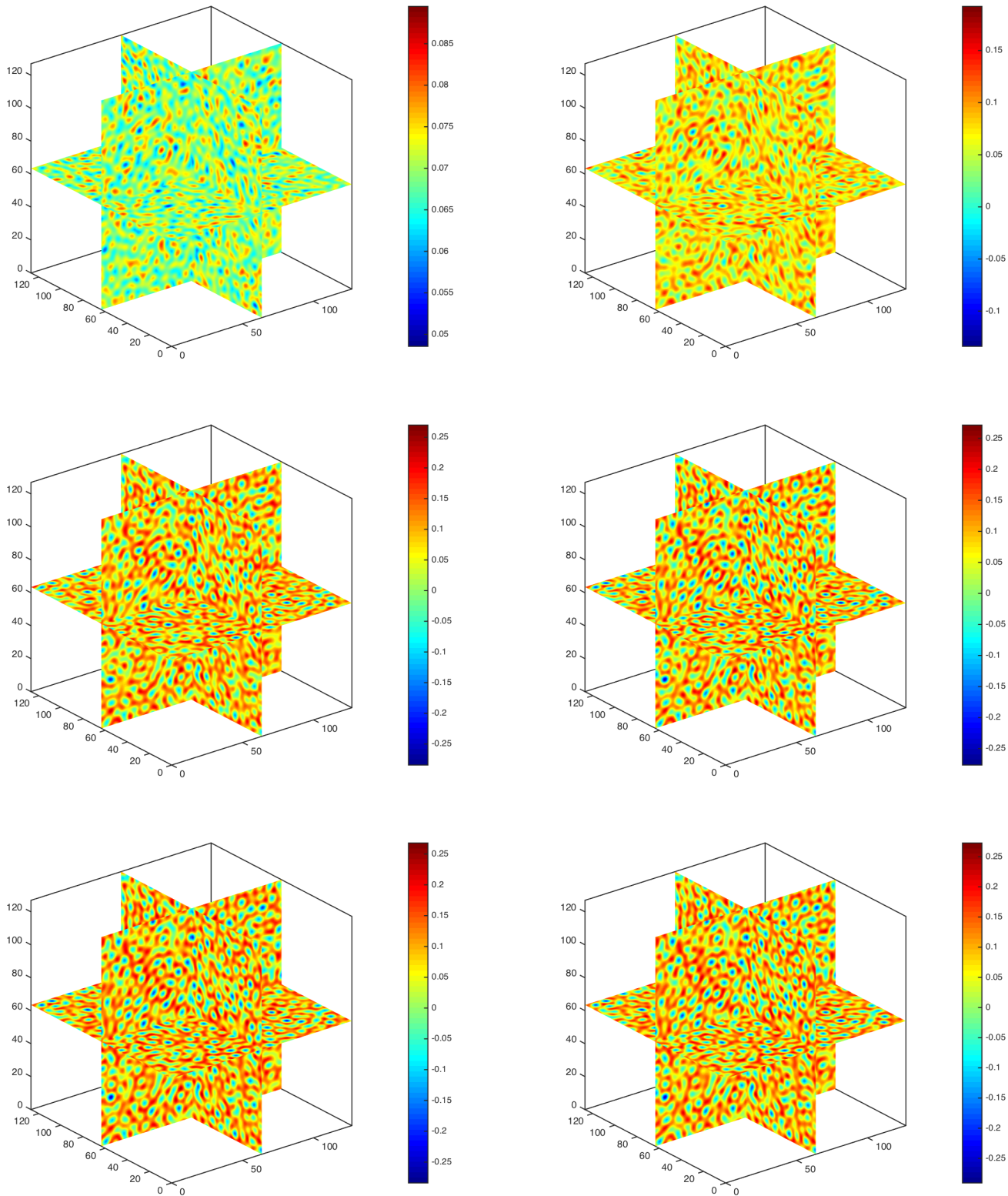

Figure 4.6. The dynamical behaviors of the phase separation behavior in 3D. Snapshots of the numerical approximation of $\phi$ are taken at $t=15,300,500,700$, 800,1000 . The computational domain is $[0,128]^{3}$. The order parameters of are $\eta=0.025, M=1, a=1.129^{3}$ Fourier modes are used to discretize the space. The time step is $\delta t=0.001$. 


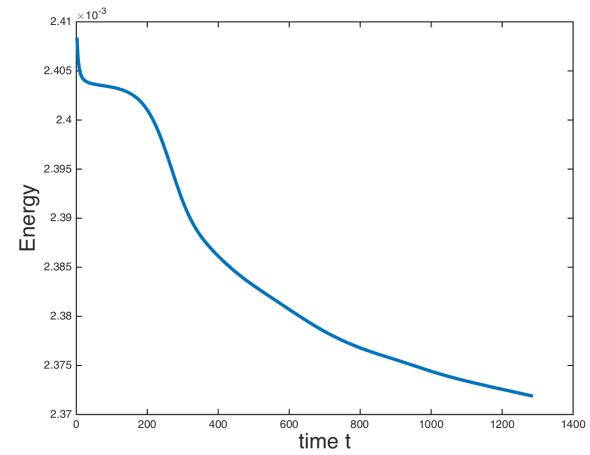

(a) $2 \mathrm{D}$

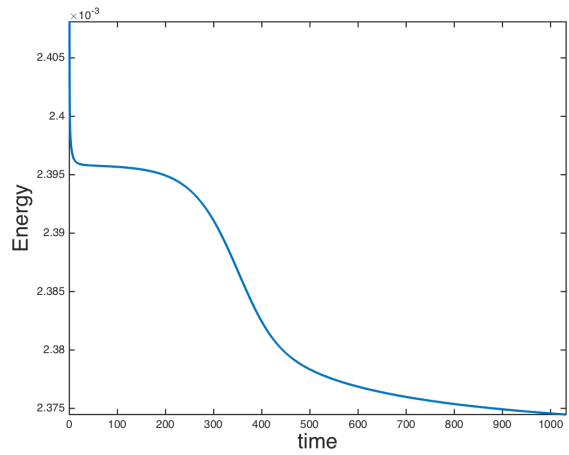

(b) $3 \mathrm{D}$

Figure 4.7. Time evolution of the free energy functional for phase transition example for $2 \mathrm{D}$ and $3 \mathrm{D}$, using the time step $\delta t=0.001$.

orientations are set inside the computational domain $[0,800]^{2}$, which leads to a complex dynamical process involving the motion of liquidcrystal interfaces and grain boundaries separating the crystals. Similar numerical examples may be found in $[7,8,31,35]$.

To define the initial configuration, we set $\phi$ to be the constant value of $\phi_{0}=0.285$. We then modify this constant configuration by setting three perfect crystallites in three small square patches of the domain as illustrated in the figure of Figure. 4.2. We use the following expression to define the crystallites:

$$
\phi\left(x_{l}, y_{l}\right)=\phi_{0}+C\left(\cos \left(\frac{q}{\sqrt{3}} y_{l}\right) \cos \left(q x_{l}\right)-0.5 \cos \left(\frac{2 q}{\sqrt{3}} y_{l}\right)\right),
$$

where $x_{l}$ and $y_{l}$ define a local system of Cartesian coordinates that is oriented with the crystallite lattice. The parameters $C$ and $q$ take the values $C=0.446$, and $q=0.66$. To generate crystallites with different orientations, we define the local coordinates $\left(x_{l}, y_{l}\right)$ using an affine transformation of the global coordinates $(x, y)$, that produces a rotation given by an angle $\theta$. We generated the three crystallites using this strategy. We took $\theta=\frac{-\pi}{4}, 0, \frac{\pi}{4}$.

We use $513^{2}$ Fourier modes to discretize the 2D space and use relatively small the time step of $\delta t=0.01$ for better accuracy. The other order parameters take the values $M=1, \eta=0.25, a=1$. Figure. 4.2 shows snapshots of the numerical solution at different computational times. We observe the growth of the crystalline phase and the motion of well-defined crystalliquid interfaces. The different alignment of the crystallites causes defects and dislocations that are clearly observed in the pictures. The solution presents similar features to those obtained in $[7,8,31,35]$. We plot the energy dissipative curve in Figure. 4.3 using four different time step of $\delta t=0.01,0.1,1,5$. We observe that the energy decreases at all times, which confirms that our algorithm is unconditionally stable, as predicted by the theory. In addition, smaller time step give much better accuracy than bigger time step. The two energy curve of $\delta t=0.01$ and 0.1 almost coincide with each other, that means the numerical solution using two time steps match well to the PDE solution. 
For 3D space, we initially set two crystallites with different orientations inside the computational domain $[0,100]^{3}$. The other order parameters are $M=1, \eta=0.5, a=1$. The initial conditions are same as (4.3). In Figure. 4.4, we observe the growth of the crystalline phase and the motion of crystalliquid interfaces from the effects of different alignments of crystallites. The solution presents similar features to those obtained in [8].

4.3. Phase transition behaviors in $\mathbf{2 D}$ and $3 \mathrm{D}$. We simulate the phase transition behavior of the PFC model. The similar numerical example can be found in $[3,35]$. With the computational domain of $[0,128]^{d}, d=2,3$, and the initial condition is $\phi(t=0)=\phi_{0}+\eta_{i, j}$, where $\phi_{0}=0.07$ and $\eta_{i, j}$ is the random number satisfying $-\phi_{0} \leq \eta_{i, j} \leq \phi_{0}$, we set $257^{2}$ Fourier modes to discretize the $2 \mathrm{D}$ space and $129^{3}$ to discretize the $3 \mathrm{D}$ space. The order parameters are $\eta=0.025, M=1$. We use time step of $\delta t=0.001$ to get the better accuracy for $2 \mathrm{D}$ and $3 \mathrm{D}$.

In Figure. 4.5 and Figure. 4.6, we show the phase transition behavior of the density field $\phi$, the results are qualitatively consistent to the simulation using the convex splitting approaches in [35]. Figure. 4.7 shows the time evolution of the energy functional for $2 \mathrm{D}$ and $3 \mathrm{D}$, respectively. We observe that the energy decreases at all times, which, again, provides numerical evidence for our method being unconditionally stable.

Remark 4.1. As the PFC model is a phase field model with a variety of distinct time scales, adaptive time stepping is highly desirable for long-time simulation. There are many adaptive time stepping algorithms available for solving this type of phase field models [2, 4, 22, 36], for instance,

$$
\delta t^{n+1}=\max \left\{\delta t_{\min }, \frac{\delta t_{\max }}{\sqrt{1+\alpha E^{\prime}\left(t_{n}\right)}}\right\},
$$

where $\delta t_{\min }$ and $\delta t_{\max }$ are smallest and largest time-steps allowed, respectively, $\alpha$ is a constant that can be adjusted in practice, and $E^{\prime}\left(t_{n}\right)=\frac{E^{n}-E^{n-1}}{\delta t^{n}}$ is the discrete time derivative of the energy (cf. [22]). The above time-adaptivity strategy can be easily incorporated in our linear schemes, we leave the details to the interested readers since the focus of this paper is to develop the linear, unconditionally energy stable schemes.

\section{Concluding Remarks}

In this paper, we have presented several efficient, semi-discrete in time, numerical schemes, provably unconditionally stable for solving the PFC model. Compared to the prevalent nonlinear schemes based on the convex splitting approaches or other nonlinear schemes, our proposed schemes conquer the inconvenience from nonlinearities by linearizing the nonlinear cubic term. We show that all three schemes developed are unconditionally energy stable. For each time step, one only needs to solve a symmetric positive definite linear system. We verify numerically that our schemes are of first and second order accuracy in time and present ample numerical results from some standard numerical tests in the context of the PFC model. 


\section{ACKNOWLEDGMENTS}

X. Yang's research is partially supported by the U.S. National Science Foundation under grant numbers DMS-1200487 and DMS-1418898, the U.S. Air Force Office of Scientific Research under grant number FA9550-12-1-0178.

\section{REFERENCES}

[1] D. M. Anderson, G. B. McFadden, and A. A. Wheeler. Diffuse-interface methods in fluid mechanics. 30:139-165, 1998.

[2] Ying Chen and Jie Shen. Efficient, adaptive energy stable schemes for the incompressible Cahn-Hilliard NavierStokes phase-field models. J. Comput. Phys., 308:40-56, 2016.

[3] M. Cheng and J.A. Warren. An efficient algorithm for solving the phase field crystal model. J. Comput. Phys., 227:6241, 2008.

[4] Andrew Christlieb, Jaylan Jones, Keith Promislow, Brian Wetton, and Mark Willoughby. High accuracy solutions to energy gradient flows from material science models. J. Comput. Phys., 257(part A):193-215, 2014.

[5] Q. Du, C. Liu, and X. Wang. A phase field approach in the numerical study of the elastic bending energy for vesicle membranes. Journal of Computational Physics, 198:450-468, 2004.

[6] K. R. Elder and Martin Grant. Modeling elastic and plastic deformations in nonequilibrium processing using phase field crystals. Phys. Rev. E., 70:051605, 2004.

[7] K. R. Elder, Mark Katakowski, Mikko Haataja, and Martin Grant. Modeling elasticity in crystal growth. Phys. Rev. Lett., 88(24):245701, 2002.

[8] Hector Gomez and Xesus Nogueira. An unconditionally energy-stable method for the phase field crystal equation. Comput. Methods Appl. Mech. Engrg., 249-252:52-61, 2012.

[9] F. Guillén-González and G. Tierra. On linear schemes for a Cahn-Hilliard diffuse interface model. J. Comput. Phys., 234:140-171, 2013.

[10] M. E. Gurtin, D. Polignone, and J. Viñals. Two-phase binary fluids and immiscible fluids described by an order parameter. Math. Models Methods Appl. Sci., 6(6):815-831, 1996.

[11] Daozhi Han. A decoupled unconditionally stable numerical scheme for the Cahn-Hilliard-Hele-Shaw system. $J$. Sci. Comput., 66(3):1102-1121, 2016.

[12] Daozhi Han and Xiaoming Wang. A second order in time, uniquely solvable, unconditionally stable numerical scheme for Cahn-Hilliard-Navier-Stokes equation. J. Comput. Phys., 290:139-156, 2015.

[13] D. Jacqmin. Calculation of two-phase Navier-Stokes flows using phase-field modeling. J. Comput. Phys., 155(1):96-127, 1999.

[14] D. Juric and G. Tryggvason. A front-tracking method for dendritic solidification. J. Comput.Phys., 123:127148, 1996.

[15] Junseok Kim. Phase-field models for multi-component fluid flows. Commun. Comput. Phys., 12(3):613-661, 2012.

[16] A. Linhananta and D. E. Sullivan. Mesomorphic polymorphism of binary mixtures of water and surfactants. Phys. Rev. E, 57:4547-4557, Apr 1998.

[17] C. Liu and J. Shen. A phase field model for the mixture of two incompressible fluids and its approximation by a Fourier-spectral method. Physica D, 179(3-4):211-228, 2003.

[18] J. Lowengrub and L. Truskinovsky. Quasi-incompressible Cahn-Hilliard fluids and topological transitions. $R$. Soc. Lond. Proc. Ser. A Math. Phys. Eng. Sci., 454(1978):2617-2654, 1998.

[19] D. I. Meiron. Boundary integral formulation of the two dimensional symmetric model of dendritic growth. Phys. D, 23:329339, 1986.

[20] M. Plapp and A. Karma. Multiscalefinite-difference-diffusion-monte-carlo method forsimulatingdendriticsolidification. J.Comput.Phys., 165:592619, 2000. 
[21] I. I. Potemkin and S. V. Panyukov. Microphase separation in correlated random copolymers: Mean-field theory and fluctuation corrections. Phys. Rev. E, 57:6902-6912, Jun 1998.

[22] Zhonghua Qiao, Zhengru Zhang, and Tao Tang. An adaptive time-stepping strategy for the molecular beam epitaxy models. SIAM J. Sci. Comput., 33(3):1395-1414, 2011.

[23] L. Rayleigh. On the theory of surface forces II. Phil. Mag., 33:209, 1892.

[24] C. Wang S. M. Wise and J. S. Lowengrub. An energy-stable and convergent finite-difference scheme for the phase field crystal equation. SIAM J. Numer. Anal., 47(3):2269-2288, 2009.

[25] Celeste Sagui and Rashmi C. Desai. Late-stage kinetics of systems with competing interactions quenched into the hexagonal phase. Phys. Rev. E, 52:2807-2821, Sep 1995.

[26] J. Shen and X. Yang. Numerical approximations of allen-cahn and cahn-hilliard equations. DCDS, Series A, 28:1169-1691, 2010.

[27] Jie Shen. Modeling and numerical approximation of two-phase incompressible flows by a phase-field approach. In Multiscale Modeling and Analysis for Materials Simulation, Lecture Note Series, Vol. 9. IMS, National University of Singapore, 2011, Edited by W. Bao and Q. Du, pages 147-196.

[28] J. Swift and P. C. Hohenberg. Hydrodynamic fluctuations at the convective instability. Phys. Rev. A, 15:319328, Jan 1977.

[29] G. Tierra and F. Guillén-González. Numerical methods for solving the Cahn-Hilliard equation and its applicability to related energy-based models. Arch. Comput. Methods Eng., 22(2):269-289, 2015.

[30] J. van der Waals. The thermodynamic theory of capillarity under the hypothesis of a continuous density variation. J. Stat. Phys., 20:197-244, 1893.

[31] C. Wang and S. M. Wise. An energy stable and convergent finite-difference scheme for the modified phase field crystal equation. SIAM J. Numer. Anal., 49:945-969, 2011.

[32] C. Xu and T. Tang. Stability analysis of large time-stepping methods for epitaxial growth models. Liq. Cryst., 44:1759-1779, 2006.

[33] X. Yang, J. J. Feng, C. Liu, and J. Shen. Numerical simulations of jet pinching-off and drop formation using an energetic variational phase-field method. J. Comput. Phys., 218(1):417-428, 2006.

[34] P. Yue, J. J. Feng, C. Liu, and J. Shen. A diffuse-interface method for simulating two-phase flows of complex fluids. J. Fluid. Mech., 515:293-317, 2004.

[35] C. Wang Z. Hu, S. M. Wise and J. S. Lowengrub. Stable and efficient finite difference nonlinear-multigrid schemes for the phase field crystal equation. J. Comput. Phys., 228:5323-5339, 2009.

[36] Zhengru Zhang, Yuan Ma, and Zhonghua Qiao. An adaptive time-stepping strategy for solving the phase field crystal model. J. Comput. Phys., 249:204-215, 2013. 